\title{
RhoA protein is generally distributed in the nuclei of cancer cells
}

\author{
YUEYING LI, YONGCHANG CHEN, YAN TAO, JIN XU and MIAO CHEN \\ School of Medical Science and Laboratory Medicine, Jiangsu University, 301 Xuefu Road, \\ Zhenjiang City, Jiangsu Province 212013, P.R. China
}

Received April 13, 2010; Accepted May 27, 2010

DOI: $10.3892 /$ or_00000948

\begin{abstract}
The aim of this study was to elucidate the localization of RhoA in human cancer tissues and cancer cell lines. Immunohistochemistry and immunofluorescence microscopy were used to determine the localization of RhoA in cancer tissues and cell lines, respectively. Western blotting was used to determine the quantity of RhoA in cell nucleus, cytosol and membrane. Immunofluorescence microscopy revealed that in all cell lines examined in this experiment (including SGC-7901, HeLa, A549 and SW480), RhoA was localized not only on the membrane, in the cytosol, but also in the nucleus. Within the nucleus its precise localization was in the nucleolus. In the transformed gastric epithelial cell line GES, RhoA was also found to be localized on the membrane, in the cytosol and the nucleus, but the nuclear location was less than that of cancer cell lines. Immunohistochemistry microscopy revealed that in cancer tissues the nuclear RhoA was obviously more than that of para-cancer tissue; in the cancer tissues undergoing inflammation and necrosis, nuclear RhoA was even higher. RhoA is generally distributed in the nucleus of cells and the distribution increases when the cells undergo tumorigenesis, suggesting a new role for RhoA.
\end{abstract}

\section{Introduction}

RhoA is the most extensively studied member of the Rho GTPase family which belongs to the Ras super family of small $\mathrm{G}$ proteins. It plays an important role in signal transduction and has been reported to regulate many biological activities including the formation of stress fibers (1), gene transcription (2), membrane transport and focal adhesions (3) and tumor progression (4).

For a long time, numerous study data (5-7) indicated that RhoA protein mainly distributes in cytoplasm and on membrane, especially in cytoplasm. However, our previous study (8) indicated that the distribution of RhoA in SGC7901 cells was not only in the membrane and cytoplasm but

Correspondence to: Dr Yongchang Chen, School of Medical Science and Laboratory Medicine, Jiangsu University, 301 Xuefu Road, Zhenjiang City, Jiangsu Province 212013, P.R. China E-mail: ycchen54@ujs.edu.cn

Key words: RhoA, distribution, nuclear, cancer tissue, cancer cell, necrosis also in the nucleus, suggesting a new role for this small $G$ protein. To study this phenomenon further, experiments were designed to detect the nuclear localization of RhoA in different cancer tissues and cancer cell lines.

\section{Materials and methods}

Cell lines. Human gastric cancer cell line SGC-7901, human cervix cancer cell line HeLa, human colon adenocarcinoma cell line SW480 and human lung cancer cell line A549 were obtained from Institute of Cell Biology of Chinese Academy of Sciences (Shanghai, China). The transformed human gastric mucosa epithelial cell line GES was a kind gift from Dr Tianji Zhou, Jiangsu University, China.

Cancer tissue samples. The cancer tissue specimens were provided by the Pathology Faculty, pertaining to First People Hospital of Jiangsu University.

Reagents. Dulbecco's modified Eagle's medium (DMEM) was from Gibco (Grand Island, NY). New-born calf serum (NBCS) was from Minhai Bio-engineering Co. (Lanzhou, China). Mouse monoclonal antibody against RhoA was from Santa Cruz Biotechnology (Santa Cruz, CA). Horseradish peroxidase (HRP)-conjugated secondary antibody was from Jackson ImmunoResearch Laboratories (West Grove, $\mathrm{PA})$. Electrochemiluminescence (ECL) reagents were from Amersham Biosciences (Buckinghamshire, UK). SABCFITC immunohistochemistry reagents were from Boster Bio-engineering Co. (Wuhan, China).

Cell culture. The cells were cultured in DMEM supplemented with $10 \% \mathrm{NBCS}$ and maintained at $37^{\circ} \mathrm{C}$ in a humidified atmosphere of $5 \% \mathrm{CO}_{2}$. The medium was changed every two days and the cells were subcultured at confluence.

Immunofluorescence microscopy. The cells grown on cover slips were fixed with freshly prepared paraformaldehyde (40 g/l in PBS) for $30 \mathrm{~min}$. After being penetrated with $30 \mathrm{ml} / 1$ Triton X-100 and blocked with $30 \mathrm{~g} / 1 \mathrm{BSA}$, the cells were incubated with the primary antibody at $4^{\circ} \mathrm{C}$ overnight $(\mathrm{o} / \mathrm{n})$ and then with fluorescein isothiocyanate (FITC) or tetrarhodamine isothiocyanate (TRITC)-conjugated second antibody for $1 \mathrm{~h}$ at room temperature (RT), with three washes after each incubation. The distribution of target protein of the cells was analyzed by fluorescence microscopy. 

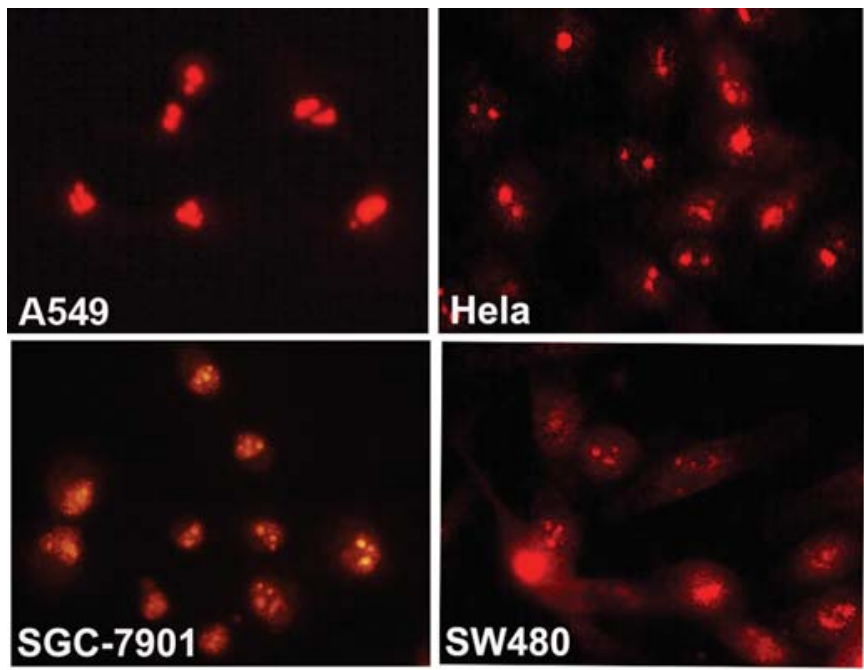

Figure 1. Nuclear localization of RhoA in different cancer cell lines. Human gastric cancer cell line SGC-7901, human cervix cancer cell line HeLa, human colon adenocarcinoma cell line SW480 and human lung cancer cell line A549 were immunofluorescently stained with a specific mAb against RhoA and the results showed that in all cell lines, RhoA was localized on the membrane, in the cytosol, and mainly in the nucleus (original magnification, $\mathrm{x} 400)$.

\section{$\mathbf{M}+\mathbf{C}$}
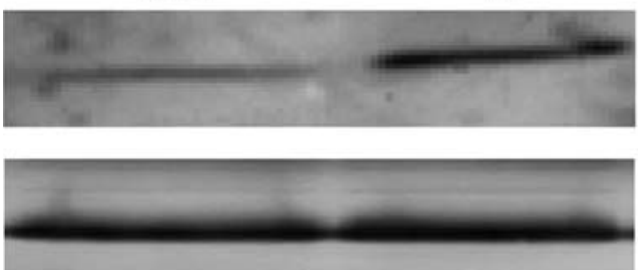

GAPDH

Figure 2. Localization of RhoA in SGC-7901 cells. SGC-7901 cells were lysed and membrane and cytosol $(\mathrm{M}+\mathrm{C})$, and nucleus $(\mathrm{N})$ fractions were obtained. Expression of RhoA in each fraction was detected by Western blotting.

Immunohistochemistry. Specimen was fixed with freshly prepared $100 \mathrm{~g} / \mathrm{l}$ paraformaldehyde, paraffin-embedded, and sliced up continuously. Every slice underwent antigen repair through heating twice in microwave oven at $92-94^{\circ} \mathrm{C}$ for $5 \mathrm{~min}$. The primary antibody was mouse monoclonal anti-RhoA and the second antibody is horseradish peroxidase (HRP)-conjugated goat anti-mouse. DAB was to show the localization of RhoA protein.

Preparation of nucleus and cytoplasm samples. According to a method descibed previously (9) cells were extracted by Dounce homogenization in HEM buffer $(10 \mathrm{mmol} / \mathrm{l}$ Hepes $\mathrm{pH}$ 7.5, $2 \mathrm{mmol} / \mathrm{l}$ EDTA, $1 \mathrm{mmol} / \mathrm{MgCl}_{2}$ ). The homogenate was centrifuged at $500 \mathrm{x} \mathrm{g}$ at $4^{\circ} \mathrm{C}$ for 5 min to obtain nuclear proteins, and the supernatant was centrifuged at $37000 \mathrm{x} g$ at $4^{\circ} \mathrm{C}$ for $30 \mathrm{~min}$. The supernatant and the pellet from the second centrifugation are referred as cytosol and membrane, respectively. The membrane preparation was washed twice with HEM buffer to remove contaminating cytosol. Protein concentrations were determined and equal amounts of protein

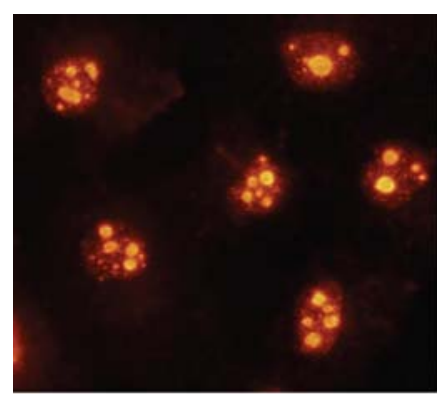

SGC-7901

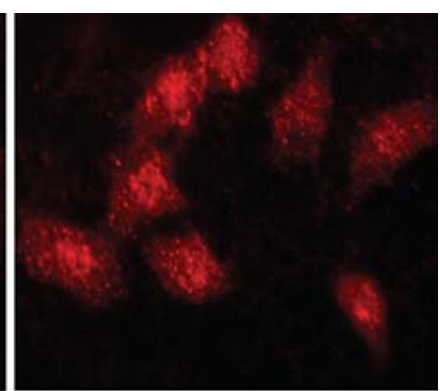

GES
Figure 3. Localization of RhoA in gastric cancer cell line SGC-7901 and transformed gastric epithelial cell line GES. RhoA was immunofluorescently stained with a specific mAb and the result showed that in both SGC-7901 and GES cells, it was localized on the membrane, in the cytosol and in the nucleus but with some discrepancy: in SGC-7901 RhoA was mainly localized in the nucleus, but in GES the localization of RhoA was almost equal in the three fractions of the cells (original magnification, $\mathrm{x} 400$ ).
A

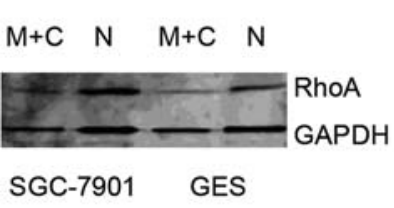

B

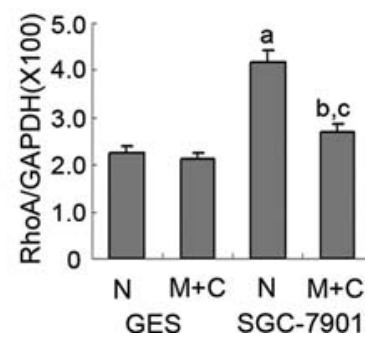

Figure 4. The expression of RhoA in SGC-7901 and GES cells. (A) Western blotting: SGC-7901 and GES cells were lysed and membrane and cytosol $(\mathrm{M}+\mathrm{C})$, and nucleus $(\mathrm{N})$ fractions were obtained. Expression of RhoA and GAPDH in each fraction was detected. (B) Western blotting results were analyzed by SPSS 13.0 Tool software and the volume ratio of RhoA/GAPDH input was calculated and presented. Each bar represents mean $\pm \mathrm{SE}$ obtained from three independent experiments. In (B), lane 1, the expression of RhoA in the nucleus of GES; lane 2, the expression of RhoA in the membrane and cytosol of GES; lane 3, the expression of RhoA in the nucleus of SGC-7901; lane 4, the expression of RhoA in the membrane and cytosol of SGC-7901. Compared with lane 1, ${ }^{\mathrm{P}} \mathrm{P}<0.001$; compared with lane 2, ${ }^{\mathrm{b}} \mathrm{P}<0.001$; compared with lane $3,{ }^{\mathrm{c}} \mathrm{P}<0.05$.

from each preparation $(30 \mu \mathrm{g})$ were subjected to SDS-PAGE/ Western blotting using mouse monoclonal anti-RhoA (1:200) and GAPDH (1:5000) antibodies.

Western blotting. Sample proteins were run on $10 \%$ SDSpolyacrylamide gels, and were blotted onto polyvinyl difluoride (PVDF) membrane. The PVDF membrane was blocked with $3 \%(\mathrm{w} / \mathrm{v})$ bovine serum albumin (BSA) in TBS-T for $1 \mathrm{~h}$ at room temperature (RT). Membranes were incubated with the primary antibody at $4{ }^{\circ} \mathrm{C} \mathrm{o} / \mathrm{n}$, and with the secondary antibody for $1 \mathrm{~h}$ at RT, with three washes after each incubation. Electrochemiluminescence reagents were used to show the positive bands on the membrane. The bands on film were analyzed with GeneSnap/Gene Tool software from Syngene (Cambridge, UK).

Statistical analysis. Data are expressed as mean \pm SE. Statistical analysis was performed using SPSS 13.0 edition 

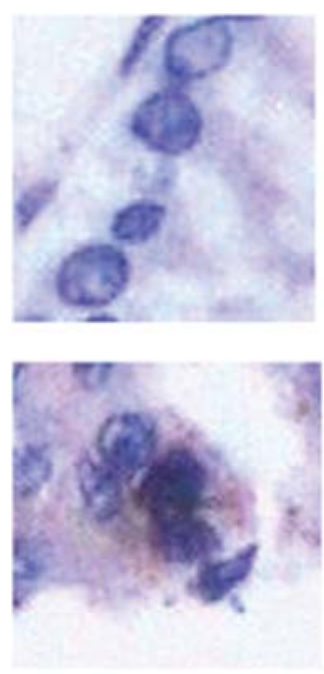

colon cancer
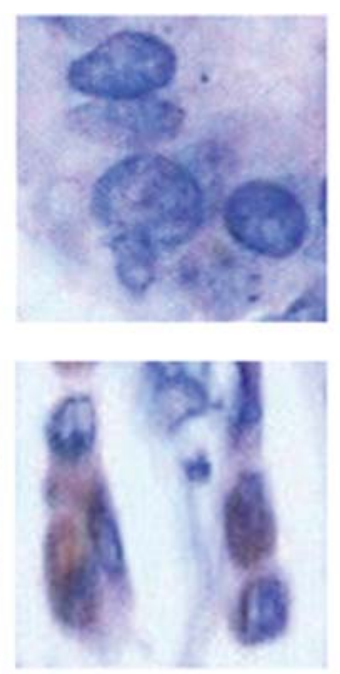

breast cancer
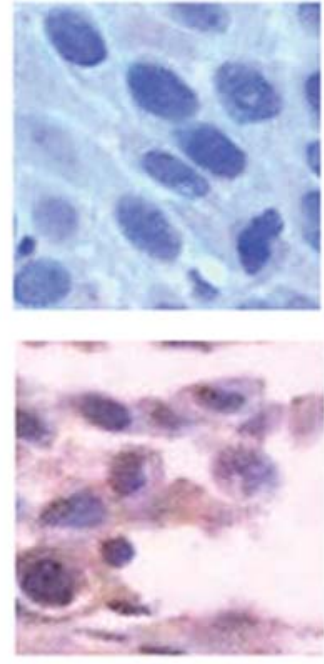

esophagus cancer

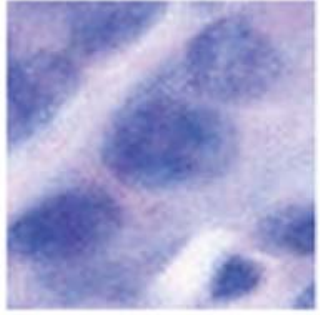

para-cancer

tissues

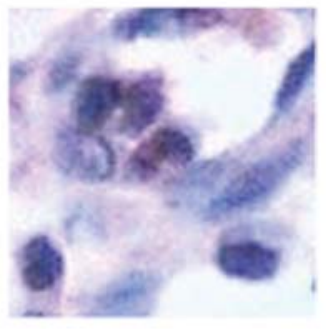

cancer

tissues

gastric cancer

Figure 5. Distribution of RhoA in different cancer tissues. RhoA was immunohistochemically stained with a specific mAb in the colon cancer, galactophore cancer, esophagus cancer and gastric cancer. The results showed that RhoA was localized in the membrane, cytosol and the nucleus of the tissues. However, RhoA in the cancer tissues was more than para-cancer tissues, especially in the nucleus (original magnification, $\mathrm{x} 400$ ).

program for ANOVA with the Scheffè multiple comparison test. Probability value at $\mathrm{P}<0.05$ was considered statistically significant.

\section{Results}

The nuclear localization of RhoA is found in different cancer cell lines. Immunofluorescence microscopy showed that in 4 cancer cell lines (SGC-7901, HeLa, A549 and SW480 cells), RhoA was localized on the membrane, in the cytosol, and mainly in the nucleus (Fig. 1). Within the nucleus its precise localization was in the nucleolus. Western blotting showed similar localization of RhoA in SGC-7901 cells (Fig. 2).

Nuclear distribution of RhoA is related to the malignancy of the cells. Results of immunofluorescence staining (Fig. 3) and Western blotting (Fig. 4) showed that SGC-7901 had more RhoA proteins than transformed gastric epithelial cell line GES. Moreover, nucleus distribution of RhoA was more than membrane and cytosol in SGC-7901. On the other hand, however, nucleus, membrane and cytosol distribution of RhoA was not obviously discrepant in GES.

RhoA is generally distributed in nuclei of cancer tissues. Immunohistochemistry staining (Fig. 5) of different cancer tissues including colon cancer, breast cancer, esophagus cancer and gastric cancer showed that the nuclear localization of RhoA was found in each type of cancer tissue. But in para-cancer tissues the nuclear distribution of RhoA was only slight.

More RhoA proteins are distributed in the nuclei of necrotizing cancer tissues. Immunohistochemistry staining (Fig. 6) of cancer tissues, para-cancer tissues, and necrotizing cancer tissues revealed that RhoA distribution in necrotizing cancer tissues was more than that of cancer tissues and paracancer tissues.

\section{Discussion}

This study detected the nuclear localization of RhoA in cancer tissues, para-cancer tissues, necrotizing cancer tissues, and several cell lines including gastric cancer cells and transformed gastric epithelial cells. The results of immunohistochemistry, immunofluorescence microscopy and Western blot analysis revealed that the nuclear localization of RhoA protein was extensive and general, with discrepancy between cancer tissues and para-cancer tissues, cancer tissues and necrotizing cancer tissues, cancer cells and transformed gastric cells, respectively. Furthermore, RhoA protein expressed excessively in cancer cells, consistent with the findings of Pan et al (10).

It is well known that RhoA protein acts as a molecular switch in the cells. Like other small guanine nucleotidebinding proteins, RhoA has GDP-bound inactive and GTPbound active forms. These two forms can be converted by GDP/GTP exchange or GTPase reactions (11). Typically, small G-proteins mainly play their role in cytoplasm and on membrane. However, recent research data showed that some small G-proteins had cytoplasma-nucleus dual distribution and the nuclear translocation of small $G$ protein was usually accompanied by the activation of the protein (12-15). For example, nuclear localized Ras protein is in GTP-bound form and this localization was possibly associated with the malignancies of the cell (15). The results of this research confirmed that cytoplasm-nuclear dual distribution of RhoA was a general phenomenon and more RhoA proteins were localized in the nuclei of cancer cells. Our previous results showed that active RhoA was the main form of this protein localized in the nucleus (8). Both of these suggested a new function of this small $\mathrm{G}$ protein. However, the role of 

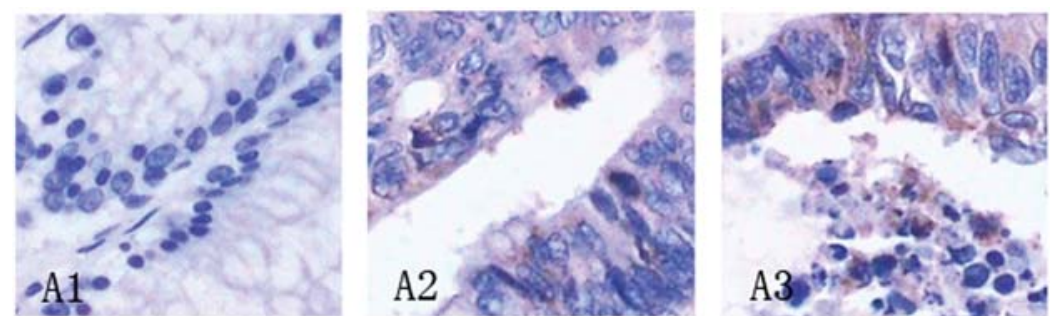

colon

cancer
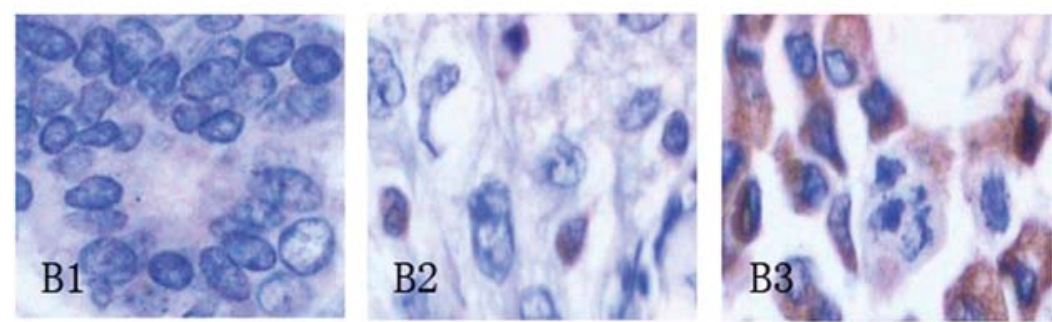

breast

cancer
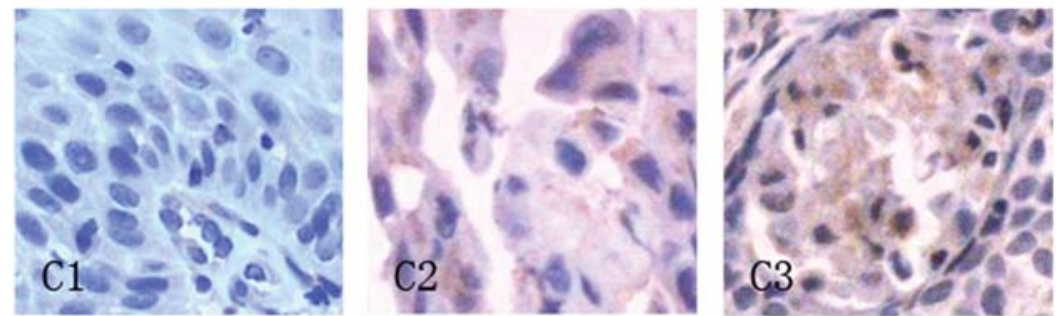

esophagus
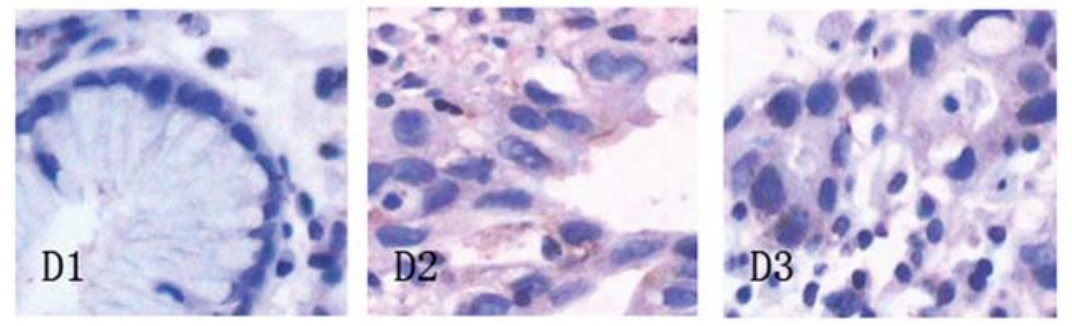

gastric

cancer

para-cancer

tissues cancer

tissues necrotizing

cancer tissues

Figure 6. Nuclear localization of RhoA in different parts of cancer tissues. RhoA was immunohistochemistrily stained with a specific mAb in the different parts of the different cancer tissues. The results showed that the necrotizing cancer tissues had more RhoA than non-necrotizing cancer tissues and para-cancer tissues, especially in the nucleus. (Original magnification, x200 and x400).

RhoA protein in the nuclear localization is still unknown. Its elucidation will be helpful to further our knowledge on the mechanisms of the migration and the invasive growth of cancer cells, and may potentially lead to novel therapeutic approaches.

Protein importing into the cell nucleus is a typical transport process between the cytosol and the nucleus. In order to enter the nucleus, proteins larger than about $60 \mathrm{kDa}$ generally require a specific nuclear localization signal (NLS) (16). Protein molecules can bind with transporting protein such as importin via NLS and get into the nucleus through an ATP-dependent process (17). However, the molecular mass of RhoA is $21 \mathrm{kDa}$ and there is no NLS in its protein structure. We suppose that the molecular mechanism of RhoA protein transportation to the nucleus was not through the classic transportation pathway, but diffusion. Related experiments are being performed in our laboratory to validate this supposition.
This study also proved that more RhoA proteins were distributed in the nuclei of necrotizing cancer tissues. Research data indicated that when the cell encounters oxidation stress damage, expression of some proteins may increase, and the proteins may be rapidly transported into the nucleus and congregate there (18). Generally, stimuli that promote protein expression are also able to promote its intracellular movement, most of them are cytoplasm-to-nucleus translocations (19). Some researchers considered that the protein into the nucleus was related to the high metabolic or low proliferative rates induced unbalance of redox state $(20,21)$. In response to stress conditions or other stimuli, such as hypoxia, hyperoxia, mechanical stress, cAMP, hypotonic media, vasoactive agents and inflammation, several cell types release ATP via either a non-lytic or a lytic mechanism from necrosis and apoptosis, reaching high local concentrations $(22,23)$. High ATP-mediated metabolic stimuli may induce quick cytoplasm to nucleus translocation of the protein. On 
the other hand, when the tissue cells undergo pathogeny body, they induce certainn inflammation factors or media, such as TNF- $\alpha$ (24). Fiemu et al (25) found that Rho inhibition might markedly decrease TNF-induced MAPK activation, at the same time proved that TNF could induce activation of upstream of ERK and P38 MAPK, and MAPK could increase the activation of GTP-binding protein Rho (25-28). It was consistent with the involvement of RhoA in TNF signaling upstream of the MAPKs. These inflammation and necrosis factors are likely to induce RhoA also into the nucleus. However, the exact mechanism is still unknown and needs further study.

According to its new localization, our results provide new insight into some unknown functions of the RhoA protein.

\section{Acknowledgements}

This study was supported by the Natural Science Foundation of Jiangsu Province, No. BK2007091; and Altitude Seminary Natural Science Research Program Item of Jiangsu Province, No. 08KJD180007.

\section{References}

1. Price LS and Collard JG: Regulation of the cytoskeleton by Rho-family GTPases: implications for tumour cell invasion. Semin Cancer Biol 11: 167-173, 2001.

2. Sotiropoulos A, Gineitis D, Copeland J and Treisman R: Signalregulated activation of serum response factor is mediated by changes in actin dynamics. Cell 98: 159-169, 1999.

3. Kjoller L and Hall A: Signaling to Rho GTPases. Exp Cell Res 253: 166-179, 1999.

4. Narumiya S: The small GTPase Rho: cellular functions and signal transduction. Biochem 120: 215-228, 1996.

5. Yonemura S, Hirao-Minakuchi K and Nishimura Y: Rho localization in cells and tissues. Exp Cell Res 295: 300-314, 2004.

6. Hoffman GR, Nassar N and Cerione RA: Structure of the Rho family GTP binding protein Cdc42 in complex with the multifunctional regulator RhoGD I. Cell 100: 345-356, 2000.

7. Adamson P, Paterson HF and Hall A: Intracellular localization of the p21rho proteins. Cell Biol 119: 617-627, 1992.

8. Yan Tao, Yong-Chang Chen and Yue-Ying Li: Localization and translocation of RhoA protein in the human gastric cancer cell line SGC-7901. World J Gastroenterol 14: 1175-1181, 2008

9. Chen JC, Zhuang S, Nguyen TH, Boss GR and Pilz RB: Oncogenic Ras leads to Rho activation by activating the mitogenactivated protein kinase pathway and decreasing RhoGTPase-activating protein activity. Biol Chem 278: 2807-2818, 2003.

10. Pan Y, Bi F, Liu N, Xue Y, Yao X, Zheng Y and Fan D: Expression of seven main Rho family members in gastric carcinoma. BBRC 315: 686-691, 2004.

11. Raftopoulou M and Hall A: Cell migration: Rho GTPases lead the way. Dev Biol 265: 23-32, 2004.
12. Moore MS and Blogel G: The GTP-binding protein Ran/TC4 is required for protein import into the nucleus. Nature 365 : 661-663, 1993.

13. Moore JD: The Ran-GTPase and cell-cycle control. Bioessays 23: 77-85, 2001.

14. Mitra RS, Zhang Z, Henson BS, Kurnit DM, Carey TE and D'Silva NJ: Rap1A and rap1B ras-family proteins are prominently expressed in the nucleus of squamous carcinomas: nuclear traslocation of GTP-bound active form. Oncogene 22: 6243-6256, 2003.

15. Wurzer G, Mosgoeller W, Chabicovsky M, Cerni C and Wesierska-Gadek J: Nuclear Ras: unexpected subcellular distribution of oncogenic forms. Cell Biochem (Suppl.) 36: $1-11,2001$

16. Moroianu J: Molecular mechanisms of nuclear protein transport. Crit Rev Eukaryot Gene Expr 7: 61-72, 1997.

17. Friedrich B, Quensel C, Sommer T, Hartmann E and Köhler M: Nuclear localization signal and protein context both mediate importin alpha specificity of nuclear import substrates. Mol Cell Biol 26: 8697-8709, 2006.

18. Wei SJ, Botero A, Hirota K, et al: Thioredoxin nuclear translocation and interaction with redox factor- 1 activates the activator protor- 1 transcription factor in response to ionizing radiation. Cancer Res 60: 6688-6695, 2000.

19. Tell G, Damante G, Caldwell D and Kelley MR: The intracellular localization of APE1/Ref-1: more than a passive phenomenon? Antioxid Redox Signal 7: 367-384, 2005.

20. Kakolyris S, Kaklamanis L, Giatromanolaki A, et al: Expression and subcellular localization of human AP endonuclease 1 (HAP1/Ref-1) protein: a basis for its role in human disease. Histopathology 33: 561-569, 1998.

21. Pines A, Perrone L, Bivi N, et al: Activation of APE1/Ref-1is dependent on reactive oxygen species generated after purinergic receptor stimulation by ATP. Nucleic Acids Res 33: 4379-4394, 2005.

22. Bowler WB, Littlewood-Evans A, Bilbe G, Gallagher JA and Dixon CJ: P2Y2 receptors are expressed by human osteoclasts of giant cell tumor but do not mediate ATP-induced bone resorption. Bone 22: 195-200, 1998.

23. Ostrom RS, Gregorian C and Insel PA: Cellular release of and response to ATP as key determinants of the set-point of signal transduction pathways. Biol Chem 275: 11735-11739, 2000.

24. Shaw PJ, Ganey PE and Roth RA: Tumor necrosis factor alpha is a proximal mediator of synergistic hepatotoxicity from trovafloxacin/lipopolysaccharide coexposure. Pharmacol Exp Ther 328: 62-68, 2009.

25. Fiemu E, Nwariaku, Rothenbach $P$, Zhu X, Turnage RH and Terada LS: Rho inhibition decreases TNF-induced endothelial MAPK activation and monolayer permeability. Appl Physiol 95: 1889-1895, 2003.

26. Chae HJ, Chae SW, Chin HY, et al: The p38 mitogen-activated protein kinase pathway regulates interleukin-6 synthesis in response to tumor necrosis factor in osteoblasts. Bone 28 : 45-53, 2001.

27. Takaishi K, Matozaki T, Nakano K and Takai Y: Multiple downstream signalling pathways from ROCK, a target molecule of Rho small $G$ protein, in reorganization of the actin cytoskeleton in Madin-Darby canine kidney cells. Genes Cells 5: 929-936, 2000.

28. Hippenstiel S, Soeth S, Kellas B, et al: Rho proteins and the p38-MAPK pathway are important mediators for LPS-induced interleukin-8 expression in human endothelial cells. Blood 95: 3044-3051, 2000. 